\title{
Our Results in the Surgical Treatment of Acute Hemorrhoidal Thrombosis
}

\author{
E.V. Ahmadova*, S.A. Aliyev, E. Aliyev, F.M. Qapaqov \\ Azerbaijan Medical University, Department of surgical disease 1, Azerbaijan, Baku
}

*Corresponding Author: Ahmadova Esmira Vaqif, Azerbaijan Medical University, Department of surgical disease 1, Azerbaijan, Baku, Tel: +994503624015; E-mail: aqaboba@mail.ru

\begin{abstract}
Hemorrhoids are common human afflictions known since the dawn of history.More than half of patients with hemorrhoids are complicated by acute thrombosis of hemorrhoids. It is unknown whether surgery is the gold standard for therapy of thrombosed. In article were described the results of surgical treatment 99 patients with acute hemorrhoidal thrombosis. All patients underwent hemorrhoidectomy during some days after hospitalization. Complications were observed at 28 patients $(28,3 \%)$, from which in early postoperative period were 25 , late complications were at 3 patients. The frequency of early and late postoperative complications is similar to those after elective hemorrhoidectomy.
\end{abstract}

Keywords: Hemorrhoid; Thrombosis; Hemorrhoidectomy; Surgery
Received Date: March 29, 2017

Accepted Date: April 21, 2017

Published Date: May 02, 2017

Citation: E.V. Ahmadova., et al. Our Results in the Surgical Treatment of Acute Hemorrhoidal Thrombosis. (2017) J Gastrointest Disord Liver Func 3(1): 98- 99.

DOI: $10.15436 / 2471-0601.17 .1448$

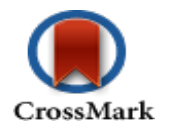

\section{Background}

Nowadays hemorrhoids remain one of the frequent proctologic disease. Analysis of patients uptake patterns for ambulatory surgical treatment shows that the vast majority of cases associated with a visit to the clinic on hemorrhoid is acute thrombosis of hemorrhoids which may recur up to 5 times a year ${ }^{[1]} .2 / 3$ of the patients seeking medical aid were the people of working age. During each exacerbation the period of temporary invalidity is from 6 to 35 days $^{[1,4]}$. Acute thrombosis of hemorrhoidal cushions can also be the primary process from which the disease begins. Despite the huge number of publications, many issues of the treatment of hemorrhoids and its complications remain argumentative.

Over the years, there are 2 opposite approaches to the treatment of this pathology. Due to the high risk of post-operative complications, the supporters of conservative tactics use medications for local (such as ointments, suppositories) and systemic exposure in acute period ${ }^{[4,7]}$. However the duration of such treatment may reach a few weeks, which doesn't correspond to modern socio-economic need of society. Palliative results of conservative therapy, the high rate of complications, as well as numerous reports on scientific validity, safety and efficiency of hemorrhoidectomy in patients with acute thrombosis of hemorrhoidal cushions in the past few years have led to the reconsider of the surgical approach in the direction of radical operative treatment ${ }^{[2]}$. We follow the practice of active surgical tactics in treatment of patients with acute hemorrhoidal thrombosis but proceed from the principle of individual approach to each patient. This feature includes the choice of the time of hemorrhoidectomy is carried out by taking into account the severity of the local inflammation.

The aim of the study: To evaluate the results of acute hemorrhoidal thrombosis in using individually-differentiated surgical tactics of the treatment.

\section{Material and Methods of Investigation}

We analyzed the results of the treatment of 99 patients with acute hemorrhoidal thrombosis of different severity that were under our observation from 2008 to 2016. There were 69 men and 30 women among them. By the moment of examination 
21 patients had acute hemorrhoidal thrombosis of the I degree of severity, 49 patients of the II degree of severity, 29 patients of the III degree of severity. All patients were operated under spinal anesthesia. Timing of the operation was carried out by taking into account the severity of the local inflammatory process. Therefore, we performed immediate, early and delayed hemorrhoidectomy. Immediate operations (within 3 days from the moment of hospitalization) were performed on 57 patients $(57,6 \%)$, with the II (43 patients) and III (14 patients) degree of severity of thrombosis. 4 of this patients were undergone open hemorrhoidectomy, 32 patients semiclosed, 21 closed hemorrhoidectomy. 21 patients had early operations (with 5 days since the day of hospitalization), 6 of them suffered from acute hemorrhoidal thrombosis of the II degree of severity, 15 of them had the III degree of severity. These patients were also performed open (6), semi-closed (13), closed (2) hemorrhoidectomy. 21 patients were undergone delayed operations who had acute hemorrhoidal thrombosis of the $1^{\text {st }}$ degree of severity. All these patients were carried out closed hemorrhoidectomy. The period of observation of the patients varied from 6 months to 5 years.

\section{Results}

Complications were observed in 28 patients $(28,3 \%)$ in postoperative period. 25 of these patients had complications in the early postoperative period, late postoperative complications were observed in 3 patients with 2 years after operation. Perianal swelling in postoperative period was observed in 4 patients (4, $04 \%$ ). These were patients who were undergone semi closed and closed hemorrhoidectomy. Postoperative complications include discharge of blood after the first defecation. 10 patients $(10 \%)$ taking part in the research complained of it. In the case of bleeding manipulations weren't required. Difficulty of defecation which required of analgesics was observed in 9 patients $(9,1 \%)$. In the late postoperative period cicatricial stricture of anal canal was observed in 2 patients, insufficiency of anal sphincter was observed in 1 patient $(1,01 \%)$. The case of recurrence of the disease wasn't observed.

According to A. Herold, P.A. Lehur, K.E. Matzel, P.R. OConnell, complications after planned hemorrhoidectomy are divided into 3 groups:

1) Occurring in $2-10 \%$ of patients severe pain, violation of defecation, bleeding, dysuria, long healing perianal wounds

2) Occurring in $1-2 \%$ of patients anal fissure, fistula, anal incontinence, anal stricture

3) Rare complications retroperitoneal infection, gangrene Fournier.

According to M. Pescatori, pain is the main complaint of patients and the reason, why they avoid operations. The rate of bleeding after hemorrhoidectomy ranges from $1 \%$ to $5 \%$. This is bleeding that requires imposing additional ligature and stitching of the node ${ }^{[6]}$. Due to date from Mauricio De La Garza 5, ${ }^{[4-7]}, 6 \%$ of patients complained of severe pains and they were relieved by narcotic analgesics ${ }^{[3]}$.
Based on the analysis of the results and published data on the results of the elective hemorrhoidectomy, it can be concluded the frequency of postoperative complications after active surgical tactics and elective hemorrhoidectomy is the same. Consequently, the possibility of early complications after hemorrhoidectomy in patients with acute hemorrhoidal thrombosis was exaggerated. As it is seen from above mentioned data, active surgical tactics in acute hemorrhoidal thrombosis don't deteriorate the results of treatment; the frequency of early and late postoperative complications is similar to those after scheduled hemorrhoidectomy. Sooner performed radical surgery promotes rapid recovery and their social and occupational rehabilitation, to avoid relapse.

\section{References}

1. Gebbensleben, O., Hilger, Y., Rohde, H. Aetiology of thrombosed external haemorrhoids: a questionnaire study. (2009) BMC Res Notes 2: S216.

Pubmed | Others

2. Janicke, D.M., Pundt, M.R., Surgical excision of symptomatic thrombosed external haemorrhoids is indicated if within 48 to 72 hours of pain onset. (1996) Emerg Med Clin North Am 14(4): S757-S788. Pubmed | Crossref| Others

3. Greenspon, J., Williams, S.B., Young, H.A., et al. Thrombosed external hemorrhoids: outcome after conservative or surgical management. (2004) Dis Colon Rectum 47: S1493-S1498.

Pubmed | Crossref| Others

4. Arezzo, A., Podzemny, V., Pescatori, M. Surgical management of hemorrhoids. State of the art. (2011) Ann Ital Chir 82(2): S163-S172. Pubmed | Others

5. Zampieri, N., Castellani, R., Andreoli, R., et al. Long-term results and quality of life in patients treated with hemorrhoidectomy using two different techniques: Ligasure versus transanal hemorrhoidal dearterialization. (2012) Am J Surg 204(5): S684-S688.

Pubmed | Crossref| Others

6. Gain, M.Y., Shakhrai, S.V., Gain, Y.M. Puncture vaccum thrombus extraction with laser coagulation of hemorrhoid node in treatment of acute external hemorrhoid thrombosis: a randomized trial. (2016) Novosti Khirurgii 24(2): S138-S145.

Others

7. Perrotti, P., Antropoli, C., Molino, D., et al. Conservative treatment of acute thrombosed external hemorrhoids with topical nifedipine. (2001) Dis Colon Rectum 44(3): S405-S409.

Pubmed |Crossref | Others 